Recepción: 20 / 04 / 2017

Aceptación: 20 / 05 / 2017

Publicación: 15 / 09 / 2017

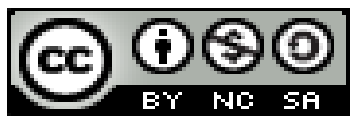

Ciencias Pedagógicas

Artículo Científico

\title{
Análisis para la elaboración de una guía didáctica para mejorar las destrezas productivas del idioma inglés dirigida a los cadetes
}

\author{
Analysis for the elaboration of a didactic guide to improve the productive skills of \\ the English language directed to the cadets
}

\begin{abstract}
Análise para o desenvolvimento de um guia didático para melhorar as habilidades produtivas da língua inglesa voltadas para cadetes
\end{abstract}

Boris E. Vasconez-Torres ${ }^{\mathrm{I}}$ bevasconez@uce.edu.ec

Gabriela M. Moso-Mena II gmmoso@uce.edu.ec

Edison S. Sanguña-Loachamin ${ }^{\text {III }}$ essanguna@uce.edu.ec

Amilcar O. Gonzalez-Diaz ${ }^{\text {IV }}$ aogonzalez@uce.edu.ec

Correspondencia: bevasconez@uce.edu.ec

I. Magister en Educación; Especialista en Currículo y Didáctica; Diploma Superior en Investigación Socioeducativa; Ingeniero en Diseño Gráfico Empresarial; Licenciado en Lingüistica Aplicada al Idioma Ingles; Licenciado en Ciencias de la Educación mención Diseño y Dibujo; Universidad Central del Ecuador, Quito, Ecuador.

II. Magister en Educación Superior y Equidad de Género; Licenciada en Ciencias de la Educacion mencion Plurilingue; Universidad Central del Ecuador, Quito, Ecuador.

III. Magister en Docencia Universitaria y Administración Educativa; Especialista en Diseño Curricular por Competencias; Licenciado en Ciencias de la Educacion mención: Plurilingue; Universidad Central del Ecuador, Quito, Ecuador.

Iv. Magister en Lingüística y Didáctica de la Enseñanza de Idiomas Extranjeros; Diploma Superior de la Enseñanza de Ingles como Segunda Lengua; Licenciado en Ciencias de la Educacion mencion Ingles; Tecnico Superior en Turismo; Universidad Central del Ecuador, Quito, Ecuador. 


\section{Resumen}

Dado que el manejo del Idioma Inglés como segunda lengua en la actualidad se ha convertido en un imperativo, la presente investigación pretende aportar con el uso de una guía didáctica que optimiza el aprendizaje de los estudiantes ayudándolos así a sentirse predispuestos a desarrollar sus destrezas lingüísticas tanto receptivas como productivas, insertándolas dentro de la metodología diaria, lo cual favorece en el rendimiento académico y la aplicación en la vida. La situación en la que viven los jóvenes, su cultura y pensamientos de cierta manera repercute en el deseo de aprender y querer practicar el idioma.

En Ecuador el Idioma Inglés ha pasado a ser parte fundamental del currículo debido a la globalización que ubica al país en un estatus importante a nivel regional y mundial, de esta manera las nuevas generaciones requieren aptitudes para desenvolverse en cualquier ámbito cultural y laboral. Los grandes adelantos en la tecnología, han hecho que los países tengan que abrir sus fronteras al intercambio global, dando como consecuencia que ese aislamiento cultural se diversifique, siendo uno de ellos las comunicaciones verbales.

Por los motivos expuestos es entonces importante la creación de una propuesta que mejore las destrezas idiomáticas del Inglés y que permita el acceso al conocimiento de los adelantos científicos y tecnológicos, de modo que el educando adquiera la mayor cantidad de datos en otro idioma a través del uso de las nuevas tecnologías de la información y la comunicación.

El interés de la presente investigación está basado en las necesidades de optimizar la enseñanza del Inglés y su proceso de aprendizaje de manera que se desarrollen talentos y conocimientos que sean aplicados en las tareas reales y permitan de alguna manera marcar la diferencia en el futuro profesional.

Palabras claves: Guía didáctica; destrezas productivas; idioma inglés. 


\section{Abstract}

Since English language management as a second language has become an imperative, the present research aims to contribute with the use of a didactic guide that optimizes students' learning, thus helping them feel predisposed to develop their language skills both receptive as well as productive, inserting them within the daily methodology, which favors academic performance and application in life. The situation in which the young people live, their culture and thoughts in a certain way has repercussions on the desire to learn and want to practice the language.

In Ecuador the English language has become a fundamental part of the curriculum due to globalization that places the country in an important status at regional and global level, so the new generations require skills to develop in any cultural and work environment. The great advances in technology have made the countries have to open their borders to global exchange, resulting in that cultural isolation is diversified, one of them being verbal communications.

For the reasons given, it is therefore important to create a proposal that improves English language skills and allows access to knowledge of scientific and technological advances, so that the learner acquires the greatest amount of data in another language through use of new information and communication technologies.

The interest of the present research is based on the needs to optimize the teaching of English and its learning process in such a way that they develop talents and knowledge that are applied in the real tasks and somehow make the difference in the professional future.

Key words: Didactic guide; productive skills; english language. 


\section{Introducción.}

Uno de los ideales en común de los países latinoamericanos es el mejoramiento de la calidad de la educación como medio de desarrollo y progreso de sus pueblos, para lo cual es primordial una reforma educativa profunda de acuerdo a la realidad del país. (Juárez N \& Comboni Salinas, 2003) (Duarte de Kendler, 2007)

En la actualidad el sistema educativo ecuatoriano está atravesando por graves problemas tanto económicos como de infraestructuras básicas, de igual forma la falta de un plan que desarrolle el nivel de la educación, hace más difícil la tarea de elevar los índices de calidad de la enseñanza y el aprendizaje. Es momento de entender que el conocimiento de la problemática educativa nacional no se lo adquiere únicamente dentro del aula de clase, sino en el quehacer de la profesión y en el estudio académico de sus diferentes estructuras: comunidad, profesorado, estudiantes, establecimientos, técnicas, métodos, planes y programas. (Richards \& Rodgers , 2001) (Brown, 2000)

Para que esto se haga realidad se deberá primeramente generar una capacitación docente elevada, que forme de manera eficaz al elemento humano, que es el sujeto motor de todo desarrollo. (Richards \& Rodgers , 2001) (Lightbown \& Spada, 1996)

El elemento humano calificado es la base indispensable para el despegue del progreso de un país. Y este elemento humano, que constituyen los educandos, es producto de un buen sistema educativo, el cual se sustenta en técnicas eficaces y en los operadores de estas técnicas, los maestros. (González, 2000) 
Anteriormente, la inexistencia de tecnología, recursos, materiales y la preparación insuficiente de docentes de diversas asignaturas, en especial en el área del idioma Inglés, que es el caso particular de este estudio, originaba que la enseñanza del mismo sea deficiente, puesto que se hacía énfasis en las estructuras gramaticales y la repetición verbal y escrita de diálogos; pero de ninguna manera se trataba de desarrollar las cuatro destrezas del idioma: leer, escribir, hablar y escuchar. (Villalobos Clavería \& Melo Hermosilla, 2008)

Se sabe que el Inglés es un Idioma internacional cuyo dominio es imperativo si se desea ser más competitivo en el ámbito profesional y laboral, "es el tercer idioma del mundo en número de hablantes que lo tienen como lengua materna: 402 millones de personas, y el segundo más hablado, detrás del chino mandarín, si se cuenta también a quienes lo tienen como segunda lengua." (Gutiérrez Ramírez \& Landeros Falcón, 2010)

La dominación cultural, económica, militar, política y científica de los Estados Unidos y del Reino Unido durante los dos últimos siglos le ha dado a la lengua inglesa el preeminente estatus como lengua franca o internacional. Por lo que, el conocimiento del idioma Inglés es prácticamente un requisito para trabajar y estudiar en las instituciones académicas internacionales.

La Escuela Superior Militar "Eloy Alfaro" (ESMIL), creada en 1895, pasó a constituirse en una institución de educación superior en 1971 mediante decreto presidencial y por ende al interior de la misma se imparten diferentes asignaturas adicionales a la preparación física de los estudiantes.

Dentro de la profesión militar en nuestro país los oficiales cumplen funciones en las que deben representar a su patria en diferentes organismos internacionales de habla inglesa como agregadurías y seminarios en los cuales deben relacionarse con extranjeros, participar en debates, 
asistir a foros e interpretar perfectamente los temas que son objeto de discusión, motivo por el cual el dominio del idioma Inglés es imprescindible.

Durante el presente año lectivo se han detectado varios problemas referentes al área de Inglés específicamente, esto se debe a que los estudiantes no han tenido un seguimiento adecuado de la asignatura, pues no se da la relevancia necesaria al idioma, que al no ser parte de la malla curricular de aprobación final, es tomado como asignatura de relleno y tiene por tanto una carga horaria limitada; de igual modo la inexistencia de estrategias metodológicas que optimicen las destrezas básicas del Inglés (Speaking, Reading, Listening, Writing); son limitantes considerables que interfieren en el proceso de aprendizaje de la segunda lengua. (Cano Lassonde, 2012)

Si la situación actual continúa de igual forma en el futuro, los educandos pueden ver muy comprometido su desempeño en las últimas instancias de educación superior así como también sus primeros años de profesionalización, debido a que la carrera militar está estrechamente relacionada con el idioma Inglés, y en la actualidad todas las áreas del conocimiento tienden a globalizar su aplicabilidad y deben ser útiles a nivel mundial y no seccional.

El estudiante debe estar preparado para una alta competitividad con un criterio más amplio, por lo que el mayor desarrollo de destrezas lingüísticas con la atención especial en la enseñanza aprendizaje del idioma Inglés le permitirá ampliar su visión y será una herramienta invaluable para su desenvolvimiento profesional.

El objetivo de la preseten investigación busca elaborar una guía didáctica que mejore las destrezas productivas del idioma Inglés dirigida a los estudiantes del Segundo Curso de la Escuela Superior Militar "Eloy Alfaro". 


\section{Materiales y métodos.}

El diagnóstico realizado a quince cadetes que pertenecen al Segundo Curso Militar paralelo "B" de la Escuela Superior Militar "Eloy Alfaro", nueve docentes del área de Inglés y seis autoridades que se desempeñan como jefes departamentales de la ESMIL, a quienes, en el proceso de investigación de la información se les aplicó encuestas diferentes, con el fin de verificar el tipo de métodos, técnicas y recursos que se aplican en el proceso de aprendizaje del idioma Inglés dentro del aula desde sus distintas perspectivas.

Los quince cadetes mencionados constituyen la población y por ende la muestra de la investigación, debido a que es un número muy bajo para realizar una técnica de muestreo. Se escogió a este curso puesto que es justamente aquí donde se ha notado la mayor cantidad de deficiencias en el rendimiento y aplicación de conocimientos de la asignatura; por tal motivo se aplicó también un test de domino del idioma, el cual vincula las cuatro destrezas básicas del idioma inglés (hablar, escuchar, escribir y leer), con el objeto de identificar cuál de ellas es la que causa mayores inconvenientes a los estudiantes.

Posteriormente se elaboró un análisis gráfico detallado de cada uno de los instrumentos utilizados para la recolección de datos con la respectiva sistematización y descripción de resultados, cuyo objetivo es mostrar las respuestas obtenidas de una forma breve y comprensible.

Al final, con los resultados obtenidos en las encuestas se realizó una triangulación diagnóstica para comparar los datos recabados, este método es el más recomendable en esta instancia puesto que son tres los estratos con los que se está desarrollando la investigación; la triangulación no es más que el cruce de información lograda, para verificar cuales son las principales semejanzas y diferencias entre los instrumentos de recolección de datos. 


\section{Resultados.}

Las encuestas realizadas a los cadetes permitieron tener conocimiento claro de los procesos de enseñanza - aprendizaje del idioma Inglés que se están utilizando en la ESMIL. El instrumento se aplicó a cadetes de Segundo Curso de Educación Militar que cursan el tercer y cuarto niveles de Inglés, para lo cual se utilizó una de las horas semanales en que se imparte la asignatura.

\begin{tabular}{|c|c|c|}
\hline Indicadores & Frecuencia & Porcentaje \\
\hline Siempre & 10 & $67 \%$ \\
\hline A veces & 3 & $20 \%$ \\
\hline Nunca & 2 & $13 \%$ \\
\hline
\end{tabular}

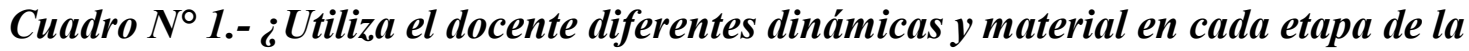
clase?

De los resultados obtenidos, se concluye que la mayoría de cadetes consideran que los profesores siempre utilizan distintas técnicas de enseñanza en las diferentes etapas de la clase.

\begin{tabular}{|c|c|c|}
\hline Indicadores & Frecuencia & Porcentaje \\
\hline Siempre & 8 & $50 \%$ \\
\hline A veces & 5 & $31 \%$ \\
\hline Nunca & 3 & $19 \%$ \\
\hline
\end{tabular}

\section{Caudro $N^{\circ} 2 .-~ ¿ C a m b i a$ el profesor su forma de impartir la materia de una clase a otra?}

En lo referente a la adopción de distintas formas de enseñanza en las diferentes horas de clase, los estudiantes opinaron en una gran mayoría que el docente está en constante cambio de las mismas. 


\begin{tabular}{|c|c|c|}
\hline Indicadores & Frecuencia & Porcentaje \\
\hline Habla & 2 & $13 \%$ \\
\hline Escribe & 3 & $20 \%$ \\
\hline Lee & 5 & $34 \%$ \\
\hline Escucha & 5 & $33 \%$ \\
\hline
\end{tabular}

Caudro $N^{\circ}$ 3.- ¿Qué destreza desarrolla más usted en el aula? Leer textos, escuchar textos, escribir mensajes o realizar exposiciones orales.

De acuerdo a la gráfica se puede observar que las destrezas productivas: hablar y escribir, tienen un desarrollo inferior a las destrezas receptivas: leer y escuchar.

\begin{tabular}{|c|c|c|}
\hline Indicadores & Frecuencia & Porcentaje \\
\hline Speaking & 4 & $27 \%$ \\
\hline Writing & 11 & $73 \%$ \\
\hline
\end{tabular}

Cuadro $N^{\circ}$ 4.- ¿De qué forma es capaz de transmitir mejor sus conocimientos del idioma Inglés a otras personas o aplicarlos en la vida práctica?

Dentro de las destrezas productivas del idioma Inglés la destreza de la escritura (writing) tiene un desarrollo superior a la destreza de la expresión oral (speaking).

\begin{tabular}{|c|c|c|}
\hline Indicadores & Frecuencia & Porcentaje \\
\hline Libros & 12 & $80 \%$ \\
\hline Proyector & 1 & $6 \%$ \\
\hline
\end{tabular}


Boris E. Vasconez-Torres; Gabriela M. Moso-Mena; Edison S. Sanguña-Loachamin; Amilcar O. Gonzalez-Diaz

\begin{tabular}{|c|c|c|}
\hline CD player & 1 & $7 \%$ \\
\hline Otros & 1 & $7 \%$ \\
\hline
\end{tabular}

Cuadro $N^{\circ}$ 5.- ¿Indique el recurso que más practica usted como estudiante en el aula?

En esta pregunta, los encuestados en un porcentaje mayoritario dicen que el recurso más utilizado por ellos son los textos de trabajos, mientras que el uso de los demás recursos tiene un porcentaje muy inferior.

\begin{tabular}{|c|c|c|}
\hline Indicadores & Frecuencia & Porcentaje \\
\hline Diaria & 0 & $0 \%$ \\
\hline Semanal & 5 & $33 \%$ \\
\hline Mensual & 9 & $60 \%$ \\
\hline Trimestral & 1 & $7 \%$ \\
\hline
\end{tabular}

Cuadro $N^{\circ}$ 6. ¿ ¿Con qué frecuencia utiliza usted el laboratorio de Inglés? Exponga para qué.

De los resultados obtenidos se puede evidenciar que los laboratorios se utilizan con muy poca frecuencia, en este caso el mayor porcentaje de utilización es de una vez por mes, y ningún cadete acude a los laboratorios de Inglés a diario.

\begin{tabular}{|c|c|c|}
\hline Indicadores & Frecuencia & Porcentaje \\
\hline Pruebas & 13 & $87 \%$ \\
\hline Exposiciones & 1 & $6 \%$ \\
\hline Tareas & 0 & $0 \%$ \\
\hline
\end{tabular}




\begin{tabular}{|l|l|l|}
\hline Participación & 1 & $7 \%$ \\
\hline
\end{tabular}

Cuadro $N^{\circ}$ 7.- ¿El tipo de evaluación a la que usted está sometido se desarrolla a través

$$
\text { de? }
$$

De acuerdo al gráfico se puede deducir claramente que los instrumentos de evaluación más utilizados son y con enorme ventaja las pruebas escritas, en contraste con los demás tipos de evaluación, los cuales se aplican en porcentajes muy inferiores.

\begin{tabular}{|c|c|c|}
\hline Indicadores & Frecuencia & Porcentaje \\
\hline Trabajo exterior & 9 & $60 \%$ \\
\hline Vida profesional & 4 & $27 \%$ \\
\hline Becas & 1 & $6 \%$ \\
\hline Otros & 1 & $7 \%$ \\
\hline
\end{tabular}

\section{Cuadro $N^{\circ} 8 .-$ ¿De qué forma cree usted que el idioma Inglés será útil en su vida profesional?}

La enorme mayoría de estudiantes considera que el dominio del idioma Inglés es altamente importante para lograr un puesto laboral en el extranjero, de igual modo un número importante sostiene que será valioso a lo largo de su vida laboral.

\begin{tabular}{|c|c|c|}
\hline Indicadores & Frecuencia & Porcentaje \\
\hline Canciones & 5 & $34 \%$ \\
\hline Dinámicas & 3 & $20 \%$ \\
\hline Videos & 5 & $33 \%$ \\
\hline Otros & 2 & $13 \%$ \\
\hline
\end{tabular}


Cuadro $N^{\circ} 9 .-$ ¿Qué tipo de actividad didáctica realiza el maestro el momento de impartir la clase?

Los resultados obtenidos concluyen en que la mayor parte de los interrogados considera que el docente motiva la clase por medio de canciones y videos, en tanto que no utiliza actividades para el desarrollo de destrezas productivas del lenguaje.

\section{Resultados del test de destrezas aplicado a los cadetes}

Posterior a la aplicación de las encuestas en los tres estratos de esta investigación, se procedió a evaluar los conocimientos de los cadetes a través de un cuestionario, en el cual se vinculan las cuatro destrezas del Inglés. Cabe resaltar que el tema general de este test es el Ecuador y sus principales características, la primera parte comprende una breve lectura y cinco preguntas acerca de la misma, luego se encuentra una sección con una pista de audio sobre de las etnias ecuatorianas, la siguiente parte está diseñada para la escritura de la información más destacada del Ecuador; finalmente un ítem para el desarrollo de la destreza verbal del estudiante.

\section{Reading}

Read the following text and answer the questions. 
Cadetes vs. Puntaje

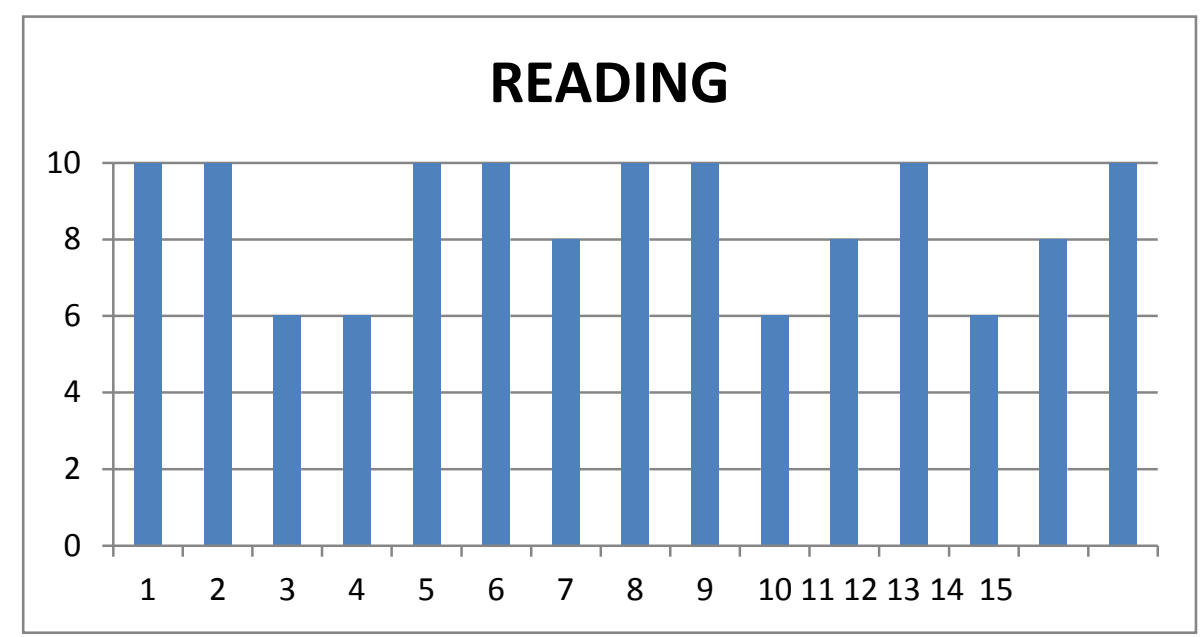

Podemos observar un rendimiento bastante alto en cuanto a la destreza de lectura en los cadetes, al realizar un promedio de los datos obtenidos encontramos que esta destreza alcanza un puntaje de $\mathbf{8 . 5 3}$ sobre 10, lo que significa un dominio importante de capacidad lectora.

\section{Listening}

Listen to someone talking about different Ecuadorians and complete the information about each person. 
Cadetes vs. Puntaje

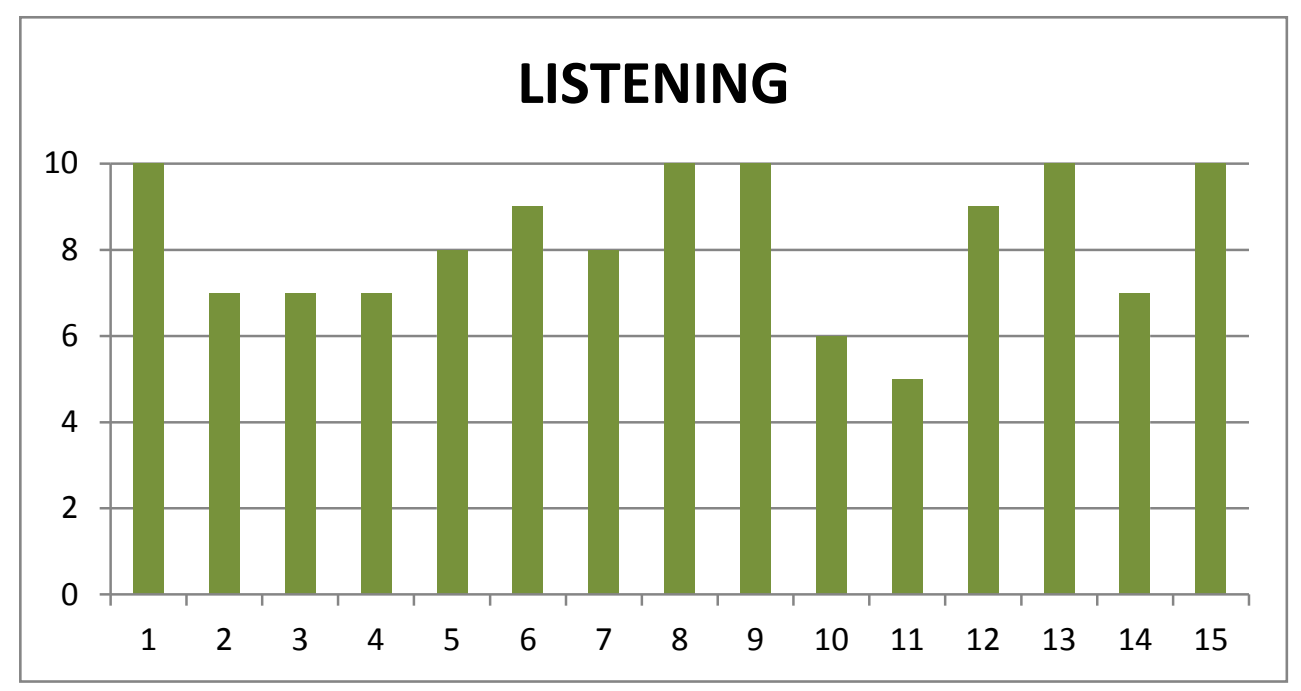

La destreza de escuchar de los cadetes nos muestra que si bien no tiene el mismo desarrollo de la destreza de leer, también obtiene un puntaje considerable de dominio, el momento de computar un promedio se obtiene un valor de $\mathbf{8 . 2}$ sobre 10 que también se considera muy aceptable.

\section{Writing}

Write a text about Ecuador. It must include three paragraphs about its most important features and sights. 
Cadetes vs. Puntaje

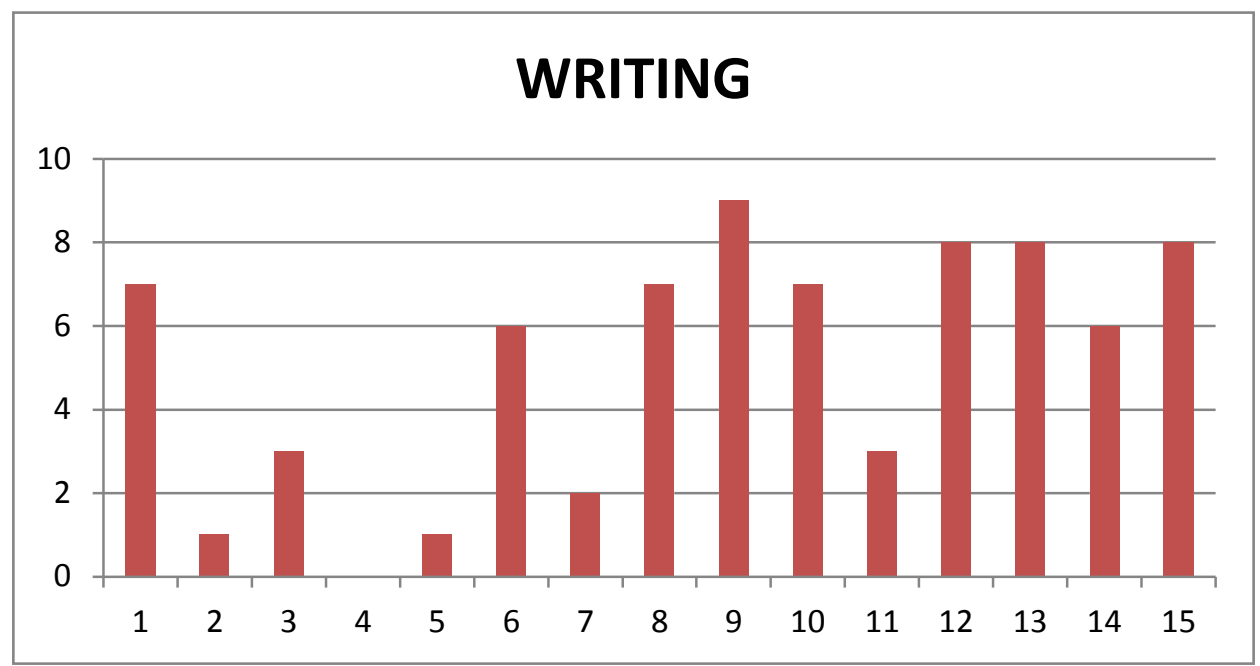

El gráfico correspondiente a la destreza de la escritura muestra que existen serias deficiencias en esta destreza, al realizar un promedio de los resultados obtenidos, se verifica que alcanza un puntaje de apenas $\mathbf{5 . 0 6}$ sobre 10, lo que muestra el limitado desarrollo de la escritura en los cadetes.

\section{Speaking}

Imagine that you are a tourist guide in Quito and you have to describe the main landmarks of the city. 
Cadetes vs. Puntaje

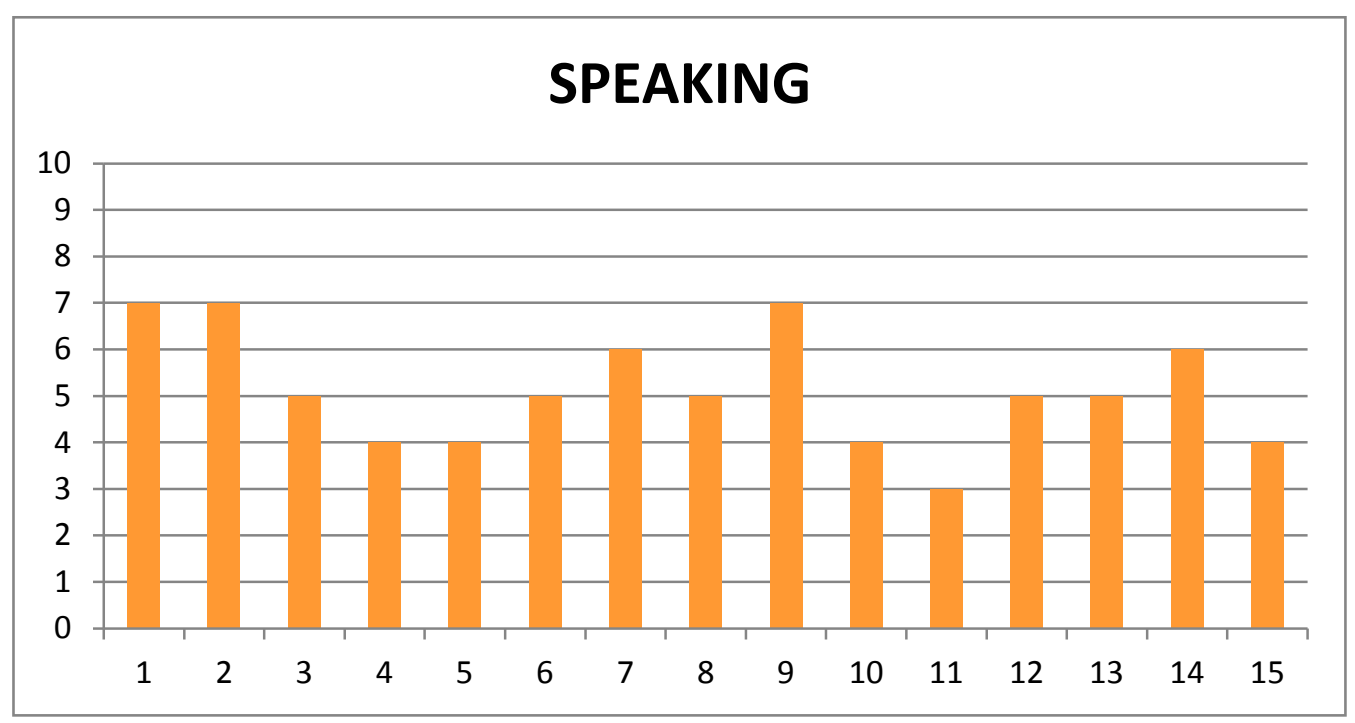

La destreza de la expresión oral de los cadetes también muestra una preocupante deficiencia, luego de haber sistematizado los datos se encuentra que el promedio de su desarrollo es de $\mathbf{5 . 2}$ sobre 10 , por lo que requiere de perfeccionamiento permanente.

Después de haber realizado este análisis de resultados se concluye que las destrezas productivas requieren mucha más atención que las destrezas receptivas; y que este desarrollo tiene que aplicarse inmediatamente si se desea optimizar las destrezas deficientes.

\section{TRIANGULACIÓN DIAGNÓSTICA}

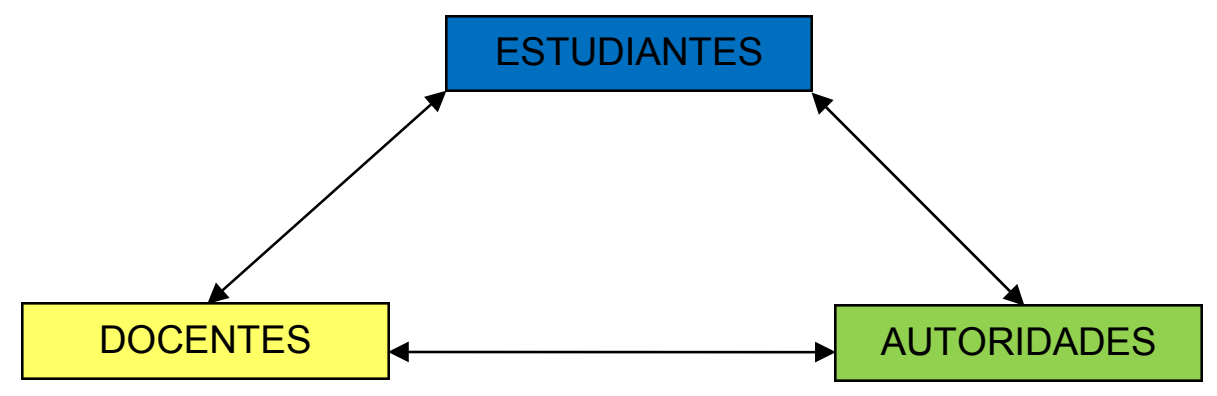


- Tanto estudiantes como docentes y autoridades están de acuerdo en que los procedimientos aplicados para las diferentes horas de clase se modifican permanentemente, con lo que se logra obtener la atención por parte de los cadetes.

- Se comprueba que en los distintos estratos del estudio se considera que el desarrollo de las destrezas receptivas del idioma es ampliamente superior al desarrollo de las destrezas productivas.

- Los tres estratos coinciden en el hecho de que el recurso más utilizado en las horas designadas para el idioma Inglés son los libros que los cadetes adquieren al inicio del año lectivo.

- La utilización del laboratorio del idioma Inglés es limitado, y esto se debe a la insuficiente carga horaria que se le asigna a la asignatura; además los temas que se abordan durante las horas en que se lo utiliza no se vinculan a temas que verdaderamente interesen al cadete en su vida profesional.

- De igual modo tanto estudiantes, docentes y autoridades consideran que el instrumento de evaluación que más se aplica para observar el avance de los conocimientos de los cadetes son pruebas, sean estas escritas u orales y que se las hace con regularidad durante el año lectivo en la asignatura.

- Finalmente se obtuvo como última evidencia el hecho de que cada estrato tiene plena seguridad en el hecho de que los métodos que se aplican para el aprendizaje del idioma Inglés están más ligados al desarrollo de destrezas receptivas (canciones, videos, lectura) y permanecen ajenos al adelanto de destrezas productivas (participación, debates, exposiciones). 


\section{Conclusiones.}

Con el fin de evidenciar el problema enunciado al inicio del proceso investigativo, este capítulo ha realizado un Diagnóstico situacional en el cual se pudo comprobar, mediante los resultados obtenidos la deficiencia en el desarrollo de las destrezas de producción del idioma Inglés, como son la escritura y la expresión oral del mismo, corroborando de este modo la necesidad de crear un instrumento didáctico que ayude de forma idónea al docente en la solución de esta problemática.

El primer instrumento para la recolección de datos durante la etapa diagnóstica fue la Encuesta, la misma que se aplicó en los tres estratos a los que se dirige este estudio. A partir de las respuestas obtenidas en las encuestas se pudo identificar cuáles eran los recursos, técnicas y destrezas que más se estaban desarrollando en la ESMIL, una vez verificada esta información se aplicó el siguiente instrumento.

Posteriormente y mediante la aplicación de un Test de Destrezas dirigido de manera exclusiva a los y las estudiantes (cadetes), se dedujo de forma categórica que el nivel de dominio de las destrezas productivas del idioma Inglés es muy deficiente y está lejos de equipararse al manejo de destrezas receptivas del lenguaje. Como paso final se realizó un cruce de información por medio de una Triangulación Diagnóstica.

El siguiente capítulo presenta la propuesta propiamente dicha, la misma que será detallada exhaustivamente y contribuirá en el proceso de optimización de las destrezas productivas del idioma Inglés y por lo tanto en el mejoramiento del proceso de enseñanza aprendizaje en la institución a la que se dirige. 


\section{Bibliografia.}

Brown, D. (2000). Principles of language learning and teaching. N.Y. USA: Longman.

Cano Lassonde, O. (2012). Antecedentes internacionales y nacionales de las tic a nivel superior: su trayectoria en panamá. Revista Electrónica “Actualidades Investigativas en Educación”, 12(3), 2-25.

Duarte de Kendler, M. (2007). Impacto de las metodologías de enseñanza utilizadas por el docente sobre la efectividad del aprendizaje del idioma inglés. Telos, 9(2), 301-317.

González, M. (2000). Lúdicas y Lenguas Extranjeras. Cómo desarrollar competencias comunicativas. Santafé de Bogotá: Cooperativa Editorial Magisterio.

Gutiérrez Ramírez, M., \& Landeros Falcón, I. (2010). Importancia del lenguaje en el contexto de la aldea global. Horizontes Educacionales, 15(1), 95-107.

Juárez N, J., \& Comboni Salinas, S. (2003). La educación en el proceso de integración de América LatinaPolítica y Cultura. Política y Cultura, 20(1), 54-77.

Lightbown, P., \& Spada, N. (1996). How Languages are Learned. New York: Oxford University Press.

Richards, \& Rodgers . (2001). Approaches and Methods in Language Teaching (2 ed.). USA: Cambridge UniversityPress.

Villalobos Clavería, A., \& Melo Hermosilla, Y. (2008). La formación del profesor universitario: Aportes para su discusión. Universidades, 39(1), 3-20. 